\title{
Reasonable doubt revisited
}

Citation for published version (APA):

Tsakas, E. (2016). Reasonable doubt revisited. Maastricht University, Graduate School of Business and Economics. GSBE Research Memoranda No. 017 https://doi.org/10.26481/umagsb.2016017

Document status and date:

Published: 01/01/2016

DOI:

10.26481/umagsb.2016017

Document Version:

Publisher's PDF, also known as Version of record

\section{Please check the document version of this publication:}

- A submitted manuscript is the version of the article upon submission and before peer-review. There can be important differences between the submitted version and the official published version of record.

People interested in the research are advised to contact the author for the final version of the publication, or visit the DOI to the publisher's website.

- The final author version and the galley proof are versions of the publication after peer review.

- The final published version features the final layout of the paper including the volume, issue and page numbers.

Link to publication

\footnotetext{
General rights rights.

- You may freely distribute the URL identifying the publication in the public portal. please follow below link for the End User Agreement:

www.umlib.nl/taverne-license

Take down policy

If you believe that this document breaches copyright please contact us at:

repository@maastrichtuniversity.nl

providing details and we will investigate your claim.
}

Copyright and moral rights for the publications made accessible in the public portal are retained by the authors and/or other copyright owners and it is a condition of accessing publications that users recognise and abide by the legal requirements associated with these

- Users may download and print one copy of any publication from the public portal for the purpose of private study or research.

- You may not further distribute the material or use it for any profit-making activity or commercial gain

If the publication is distributed under the terms of Article $25 \mathrm{fa}$ of the Dutch Copyright Act, indicated by the "Taverne" license above, 


\section{Maastricht University}

Elias Tsakas

Reasonable doubt revisited

RM/16/017

\section{GSBE}

Maastricht University School of Business and Economics

Graduate School of Business and Economics

P.O Box 616

NL- 6200 MD Maastricht

The Netherlands 


\title{
Reasonable doubt revisited
}

\author{
ELIAS TSAKAS* \\ Maastricht University
}

March 31, 2016

\begin{abstract}
According to the common definition, the standard of reasonable doubt is a threshold such that, the defendant is convicted if and only if the probability that the juror attaches to the defendant being guilty is above this threshold. In this paper we prove that generically such a threshold exists if and only if the juror reasons only about the defendant's guilt and nothing else. We discuss the implications of this result, and subsequently we propose a weakening of the aforementioned definition, by substituting the standard of reasonable doubt with a pair of standards, an upper and a lower one, thus obtaining a sufficient condition for conviction and a sufficient condition for acquittal. Finally, we prove that the lower standard always exists, whereas the upper standard exists if and only if the juror prefers to always convict a guilty defendant, irrespective of the circumstances.
\end{abstract}

\section{Introduction}

Modelling court decisions has been a fundamental issue in the literature on economics of crime (e.g., Becker, 1968; Andreoni, 1991; Friedman and Wickelgren, 2006; Persson and Siven, 2007), as well as on voting rules in (jury) committees (e.g., Feddersen and Pesendorfer, 1998; Gerardi, 2000). The most central concept in these literatures is that of the standard of reasonable doubt, which is defined as the probability threshold for convicting/acquitting the defendant. ${ }^{1}$ Actually, the relevance of this notion is not limited to court decisions, but is also extended to a much larger family of binary decisions. For instance, the decision to treat a patient with a specific drug often depends on whether

${ }^{*}$ Department of Economics (AE1), Maastricht University, P.O. Box 616, 6200 MD, Maastricht, The Netherlands; Tel: +31-43-38 83649; E-mail: e.tsakas@maastrichtuniversity.nl

${ }^{1}$ For an overview of the jury system, we refer to Jonakait (2003). 
the probability of success is above or below some threshold. Likewise, the decision to adopt a policy often depends on whether the probability of this policy solving a specific problem is above or below some threshold. In this sense, choice rules based on the concept of reasonable doubt, appear in a wide number of fields, including law, economics, political science, health sciences, etc.

The main question that arises in relation to the notion of reasonable doubt is whether we should formally define it. For instance, within the law literature there is a longstanding debate on this matter (e.g., Tribe, 1971; Diamond, 1990; Mulrine, 1997; Lillquist, 2002). In fact, several scholars are in general against the use of decision-theoretic models for court decisions (Tribe, 1971). Part of the argumentation against formally defining a standard of reasonable doubt is based on the fact that empirical evidence is far from being conclusive (see Section 5.1).

The aim of this paper is to provide a conclusive answer to this question using a general decisiontheoretic model. ${ }^{2}$ Our conclusion turns out to be quite surprising. In particular, our main (impossibility) result proves that a standard of reasonable doubt exists if and only if the only relevant event (from the juror's point of view) is the defendant's guilt. That is, if the juror reasons about other events - such as for instance the defendant's intention to commit the crime - there is no threshold probability of guilt, above which (resp., below which) the juror would convict (resp., acquit) the defendant. Note that our result is very robust, in the sense that it does not depend on which this event could be, as long as it is relevant to the case. In fact, since it is hard to imagine of jurors who disregard every event besides the defendant's guilt, we can conclude that a standard of reasonable doubt does not even exist.

The implications of the previous result are twofold. On the one hand, we strongly reinforce the view that a standard of reasonable doubt should not be explicitly defined, even in ideal cases where the juror's preferences are known. However, this conclusion is not based on arguments put forth by those opposed to a formal definition of reasonable doubt, but rather on the mere fact that the standard of reasonable doubt is not well-defined, unless we impose the very restrictive assumption that the juror reasons only about the defendant's guilt and nothing more. ${ }^{3}$ Secondly, our result implies that the extensive literature - on economics of crime or jury decisions - that uses reasonable doubt as a choice rule, implicitly imposes the aforementioned restrictive assumption. In this sense, some of the obtained results may have to be revisited.

In the second part of the paper, we attempt to partially resolve the impossibility result, by weakening the definition of reasonable doubt. In particular, instead of considering a (unique)standard

\footnotetext{
${ }^{2}$ Throughout the paper, we study the concept of reasonable doubt in the context of court decisions, even though as we have already mentioned - our framework can also be used to model other types of decisions.

${ }^{3}$ In fact, the irony is that, using decision-theoretic tools, we reinforce the view of some scholars who are against the use of decision theory for studying court decisions.
} 
such that the defendant is convicted if and only the probability of being guilty is above this standard, we define an upper and a lower standard, such that the defendant is convicted (resp., acquitted) if the probability of guilt is above the upper standard (resp., below the lower standard). In this sense, we provide a sufficient condition for conviction and a sufficient condition for acquittal. Obviously, a standard of reasonable doubt exists if and only if the upper threshold coincides with the lower one. Then, we move on to prove that the lower standard of reasonable always exists. On the other hand, the upper standard exists if and only if the juror prefers to convict a guilty defendant irrespective of the circumstances. This implies that a sufficient condition for conviction may not exist if there are extenuating circumstances, e.g., this is the case if the juror prefers to acquit a guilty defendant who committed the crime unintentionally, over convicting him.

The paper is structured as follows: In Section 2 we introduce our framework and define the standard of reasonable doubt. Section 3 contains our main impossibility result. In Section 4 we define the weak standard of reasonable doubt and we prove our positive result. Section 5 contains a discussion. All proofs are relegated to the Appendix.

\section{Definition of reasonable doubt}

There are two agents, a (female) juror and a (male) defendant. Let $(\Omega, \mathcal{E})$ be a measurable state space. Each state $\omega \in \Omega$ is a full description of all relevant aspects of the world, implying that if the state is known all uncertainty - about both past and future events of interest - is resolved. Each subset in the $\sigma$-algebra $\mathcal{E}$ is an event. Let $G \in \mathcal{E}$ be the event that the defendant is guilty of the crime he is accused for, with the complement $I:=\neg G$ denoting the event that he is innocent. Note that $G$ is a coarse description of the world, in the sense that it contains different specifications of how the crime could have taken place, e.g., there are different ways of committing a crime, differing for instance in the defendant's intention to commit the crime or in the degree of cruelty involved. In either case, we assume that the law is detailed enough to clearly specify at which states the defendant is considered guilty (resp., innocent). Thus, the events $G$ and $I$ have a well-defined interpretation in the natural language.

A finite algebra $\mathcal{F} \subseteq \mathcal{E}$ of subsets of $\Omega$ is called a frame, and contains the events that the juror reasons about. Events outside $\mathcal{F}$ are not even considered by the juror, either because she is unaware of them or because she consciously disregards them. We define the frame $\mathcal{R} \subseteq \mathcal{E}$ to be the collection

of events that the juror reasons about at the time of her decision. Actually, $\mathcal{R}$ could be the outcome of some reasoning process undertaken by the juror, thus implying that the juror may consider different frames throughout the trial, before she eventually ends up reasoning about the events in $\mathcal{R}$. This 
reasoning process is not explicitly modelled, and we focus directly on its outcome, i.e., on the frame $\mathcal{R}$. We naturally assume that the event $G$ is always $\mathcal{R}$-measurable, and a fortiori so is the event $I$, i.e., the juror always reasons about the defendant's guilt/innocence. In fact, if she reasons only about $G$, her algebra $\mathcal{R}$ will be $\mathcal{G}:=\{\Omega, G, I, \emptyset\}$. Let $\mathcal{P} \subseteq \mathcal{R}$ denote the partition of $\Omega$ that generates $\mathcal{R}$. ${ }^{4}$

Let $X \subseteq[0, \infty]$ be the set of all possible decisions that the juror could take according to the law. ${ }^{5}$ Throughout the paper we naturally assume that $0 \in X$, i.e., acquitting the defendant is one of the possible outcomes. For notation simplicity we define $X_{+}:=X \backslash\{0\}$. The juror has an $\mathcal{R}$-measurable state-dependent utility function $U: \Omega \rightarrow \mathbb{R}^{X}$ and forms a subjective belief $\pi \in \Delta(\Omega, \mathcal{R}),{ }^{6}$ such that her preferences are represented by the state-dependent expected utility (SDEU) function,

$$
\mathbb{E}_{\pi} U(x)=\int_{\Omega} U_{\omega}(x) d \pi(\omega) .
$$

There are various axiomatizations of state-dependent expected utility functions in the literature, both within the Savage and the Anscombe-Aumann framework (Fishburn, 1973; Karni et al., 1983; Karni, 1993a,b).

Before moving forward, let us elaborate on how our SDEU function emerges. First, we fix the frame $\mathcal{R}$. Second, we fix the $\mathcal{R}$-measurable state-dependent utility function $U$, which represents the juror's preferences (over the set of $\mathcal{R}$-measurable acts) at the time of the decision. We allow her preferences to be frame-dependent. ${ }^{7}$ In this sense, our model is rich enough to capture framing effects. While the utility function may vary across frames, it is still stable, in the sense that it is specified for every given frame before the juror enters the courtroom. In other words, changes in the utility function during the trial can occur only due to changes in the frame that she considers. The latter implies that we could also think of the juror's utility function (given an arbitrary fixed frame) as some ideal (frame-dependent) social welfare function carried by the representative ideal juror (under this frame). Thus, the utility function could in principle even have a normative interpretation, even though we do not postulate that this is necessarily the case. ${ }^{8}$

Finally, the juror forms a belief $\pi$ about the events in $\mathcal{R}$. Once again, the juror's belief may

\footnotetext{
${ }^{4}$ The $\sigma$-algebra generated by a collection of events $\mathcal{Q} \subseteq \mathcal{E}$ is denoted by $\sigma(\mathcal{Q})$ and is the smallest $\sigma$-algebra containing all subsets in $\mathcal{Q}$. For a given finite algebra $\mathcal{R}$, there exists a unique (finite) partition $\mathcal{P}$ such that $\mathcal{R}=\sigma(\mathcal{P})$, e.g., $\mathcal{G}=\sigma(\{G, I\})$.

${ }^{5}$ It may be sometimes convenient to interpret $\infty$ as the death penalty, though we do not need to do so for the purposes of this paper.

${ }^{6}$ As usual, $\mathbb{R}^{X}$ denotes the set of all functions from $X$ to $\mathbb{R}$, while $\Delta(\Omega, \mathcal{R})$ denotes the set of all probability measures over the measurable space $(\Omega, \mathcal{R})$.

${ }^{7}$ Ahn and Ergin (2010) also consider preferences that vary across different frames. However, their model differs from ours in that their utility function does not depend on the frame. Instead, differences in the preferences across different frames are reflected only on the juror's subjective beliefs.

${ }^{8}$ For a detailed discussion on the normative nature of the juror's utility function, see Lillquist (2002).
} 
change during the trial. This can be either due to changes in the frame that she considers, or due to updating given a fixed frame. In any case, such changes are part of her reasoning process, which - as we have already mentioned - we do not model here. ${ }^{9}$ Unlike the utility function, the juror's beliefs have no normative side, i.e., there is no restriction on what the juror should believe. Overall, the probability space $(\Omega, \mathcal{R}, \pi)$ reflects the juror's subjective assessment of the situation at the time of her decision.

Since $U$ is $\mathcal{R}$-measurable, for each $P \in \mathcal{P}$ there exists some $U_{P} \in \mathbb{R}^{X}$ such that $U_{\omega}=U_{P}$ for all $\omega \in P$. This also implies that Eq. (1) can be rewritten as

$$
\mathbb{E}_{\pi} U(x)=\sum_{P \in \mathcal{P}} \pi(P) U_{P}(x)
$$

Example 1. Let $\Omega=\left\{\omega_{1}, \omega_{2}, \omega_{3}\right\}$ and $\mathcal{E}=2^{\Omega}$. Moreover, let $G=\left\{\omega_{1}, \omega_{2}\right\}$ and assume that the juror reasons only about the defendant's guilt/innocence, i.e., $\mathcal{R}=\mathcal{G}=\left\{\Omega,\left\{\omega_{1}, \omega_{2}\right\},\left\{\omega_{3}\right\}\right.$, $\}$. In fact the two states in $G$ differ in the defendant's intention to commit the crime, viz., at $\omega_{1}$ the defendant committed the crime unintentionally, whereas at $\omega_{2}$ he did it intentionally. However, the juror does not reason about the defendant's intentions, i.e., $\left\{\omega_{2}\right\} \notin \mathcal{R}$, and therefore she does not distinguish between $\omega_{1}$ and $\omega_{2}$. Then, the juror's state-dependent utility function $U_{\omega}: X \rightarrow \mathbb{R}$ is such that $U_{\omega_{1}}=U_{\omega_{2}}$. Thus, we define $U_{G}:=U_{\omega_{1}}=U_{\omega_{2}}$ and $U_{I}:=U_{\omega_{3}}$. The rough interpretation is obvious: $U_{G}$ (resp., $U_{I}$ ) is the juror's utility whenever the defendant is guilty (resp., innocent). This framework, with $\mathcal{R}=\mathcal{G}$, is the one considered by most papers in the literature (e.g., Andreoni, 1991; Connolly, 1987; Friedman and Wickelgren, 2006).

Throughout the paper we impose the following natural assumption.

Assumption 1. For every $x \in X_{+}$, we let $U_{\omega}(x)<U_{\omega}(0)$ for every $\omega \in I$.

The interpretation is straightforward, viz., the juror prefers acquitting an innocent defendant over convicting him, irrespective of the circumstances or the magnitude of the sentence. Notice that we do not require the juror to necessarily prefer convicting a guilty defendant, as this may depend on the precise circumstances under which the crime was committed or on the magnitude of $x$.

According to the standard definition, from the juror's point of view the defendant is guilty beyond reasonable doubt whenever the probability $\pi(G)$ that she attaches to the defendant being guilty is

\footnotetext{
${ }^{9}$ Reasoning processes that fit our framework are the ones of existing models of rational inattention (De Oliveira et al., 2016) or costly contemplation (Ergin and Sarver, 2010), where the juror chooses how much (costly) effort to exert into resolving her subjective uncertainty about her beliefs or her preferences respectively. Another possibility, also consistent with our setting, consists of the models of persuasion, where the prosecutor decides how much effort to put into collecting evidence, and the juror's belief is a noisy function of the collected evidence (Kamenica and Gentzkow, $2011,2014)$.
} 
larger than some fixed threshold. ${ }^{10}$ We will often refer to this threshold as the standard of reasonable doubt.

Let us introduce some additional notation and terminology. For an arbitrary $\mathcal{R}$-measurable $U: \Omega \rightarrow \mathbb{R}^{X}$ and a given $x \in X_{+}$, define the $\mathcal{R}$-measurable function $V(x): \Omega \rightarrow \mathbb{R}$ by $V_{\omega}(x):=$ $U_{\omega}(x)-U_{\omega}(0)$, and we naturally obtain $V_{P}(x):=U_{P}(x)-U_{P}(0)$ and $\mathbb{E}_{\pi} V(x):=\mathbb{E}_{\pi} U(x)-\mathbb{E}_{\pi} U(0)$ for an arbitrary $\pi \in \Delta(\Omega, \mathcal{R})$. Then, we define the (compact and convex) subsets of $\Delta(\Omega, \mathcal{R})$,

$$
\begin{aligned}
& C_{x}:=\left\{\pi \in \Delta(\Omega, \mathcal{R}): \mathbb{E}_{\pi} V(x) \geq 0\right\} \\
& D_{p}:=\{\pi \in \Delta(\Omega, \mathcal{R}): \pi(G) \geq p\}
\end{aligned}
$$

where $C_{x}$ is interpreted as the set of beliefs that make the juror weakly prefer to convict the defendant, and $D_{p}$ is interpreted as the set of beliefs that put probability at least $p$ to the defendant being guilty.

Definition 1. Fix an arbitrary $\mathcal{R}$-measurable $U: \Omega \rightarrow \mathbb{R}^{X}$ and an arbitrary $x \in X_{+}$. Then, we say that $p_{x} \in \mathbb{R}_{+}$satisfies the reasonable doubt criterion for the sentence $x \in X_{+}$if $C_{x}=D_{p_{x}}$.

In other words, $p_{x}$ satisfies the reasonable doubt criterion for $x \in X_{+}$whenever

$$
\mathbb{E}_{\pi} U(x) \geq \mathbb{E}_{\pi} U(0) \Leftrightarrow \pi(G) \geq p_{x}
$$

i.e., a juror (weakly) prefers to convict the defendant if and only if the probability she attaches to the defendant being guilty is at least equal to $p_{x}$. This definition is standard in the literature (e.g., Andreoni, 1991; Feddersen and Pesendorfer, 1998). Note that the standard of reasonable is subjective, only as long as $U$ represents the juror's own preferences. If we instead adopt the normative interpretation of $U$ being some ideal social choice function, the standard of reasonable doubt becomes objective.

Example 1 (cont). Recall our example with $\Omega=\left\{\omega_{1}, \omega_{2}, \omega_{3}\right\}, G=\left\{\omega_{1}, \omega_{2}\right\}$ and $\mathcal{R}=\mathcal{G}$. Let $U_{G}(x)=-x^{2}+2 x-1$ and $U_{I}(x)=-x+1$. Now, take the sentence $x=1$, and observe that

$$
\begin{aligned}
C_{1} & =\left\{\pi \in \Delta(\Omega, \mathcal{R}): \pi(G) U_{G}(1)+(1-\pi(G)) U_{I}(1) \geq \pi(G) U_{G}(0)+(1-\pi(G)) U_{I}(0)\right\} \\
& =\{\pi \in \Delta(\Omega, \mathcal{R}): \pi(G) \geq 1 / 2\} \\
& =D_{1 / 2},
\end{aligned}
$$

thus implying that $p_{1}=1 / 2$ satisfies the reasonable doubt criterion.

The sentence $x \in X_{+}$is said to be trivial if either $V(x) \geq 0$ or $V(x) \leq 0 .{ }^{11}$ It is nontrivial otherwise. Note that if we impose Assumption 1, it cannot be the case that $V(x) \geq 0$, and therefore $x \in X_{+}$is nontrivial if and only if there exists some $\omega \in G$ such that $U_{\omega}(x)>U_{\omega}(0)$.

\footnotetext{
${ }^{10}$ Obviously, $\pi(G)$ is well-defined, since $G \in \mathcal{R}$ and $\pi \in \Delta(X, \mathcal{R})$.

${ }^{11}$ For an arbitrary $Y: \Omega \rightarrow \mathbb{R}$, we write $Y \geq 0$ whenever $Y(\omega) \geq 0$ for all $\omega \in \Omega$.
} 
Proposition 1. Let $p_{x} \in \mathbb{R}_{+}$satisfy the reasonable doubt criterion for $x \in X_{+}$. Then,

(i) $x$ is nontrivial if and only if $p_{x} \in(0,1)$,

(ii) if $x$ is nontrivial, then $p_{x}$ is unique.

Throughout the rest of the paper, if there exists some $p_{x}$ satisfying the reasonable doubt criterion, we refer to it as the standard of reasonable doubt. In the next section, we investigate the conditions under which a standard of reasonable doubt exists.

\section{Existence of standard of reasonable doubt}

So far, we have defined the standard of reasonable doubt, but we have not answered the most fundamental question, viz., does it always exist? It turns out that this is possible only under very stringent conditions.

Main (Impossibility) Theorem. Fix a nontrivial $x \in X_{+}$. Then, there exists some $p_{x} \in(0,1)$ satisfying the reasonable doubt criterion if and only if $V(x): \Omega \rightarrow \mathbb{R}$ is $\mathcal{G}$-measurable.

The main conclusion of the previous result is strong and surprising. It essentially says that a standard of reasonable doubt does not even exist, unless either (i) the juror reasons only about the defendant's guilt/innocence and nothing else, or (ii) she reasons about additional events but does not find them relevant. Let us illustrate such a case with an example.

Example 2. Let $\Omega=\left\{\omega_{1}, \omega_{2}, \omega_{3}\right\}$, with $G=\left\{\omega_{1}, \omega_{2}\right\}$ and $F=\left\{\omega_{2}\right\}$ denoting the event that the defendant is guilty and the event that he intended to commit the crime respectively. Moreover, assume that the juror reasons about every event, i.e., $\mathcal{R}=\sigma(\{F, G\})=2^{\Omega}$, thus implying that $\Delta(\Omega, \mathcal{R})=\Delta(\Omega)$. Furthermore, let the juror's state-dependent utility function be such that $U_{\omega_{1}}(x)=$ $-x^{2}+4 x, U_{\omega_{2}}(x)=-3.5 x^{2}+4 x$ and $U_{\omega_{3}}(x)=-x+1$. Take the sentence $x=1$, and observe that

$$
\begin{aligned}
C_{1} & =\left\{\pi \in \Delta(\Omega): 4 \pi\left(\omega_{1}\right)+1.5 \pi\left(\omega_{2}\right) \geq 1\right\} \\
& \neq\left\{\pi \in \Delta(\Omega): \pi\left(\omega_{1}\right)+\pi\left(\omega_{2}\right) \geq p\right\} \\
& =D_{p}
\end{aligned}
$$

for all $p \in \mathbb{R}_{+}$, thus implying that there is no standard of reasonable doubt. Of course, this is obvious given our Impossibility Theorem, since $U(1)$ is not $\mathcal{G}$-measurable, viz., $U_{\omega_{1}}(1) \neq U_{\omega_{2}}(1)$. Let us illustrate graphically why this is the case. First, note that the shaded area contains to the beliefs in $C_{1}=\left\{\pi \in \Delta(\Omega): \mathbb{E}_{\pi} V(1) \geq 0\right\}$. The area above the dashed linear segment contains the beliefs in 


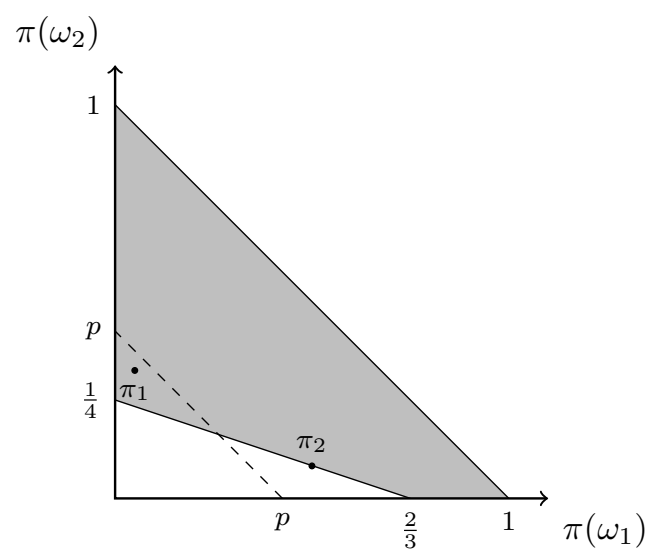

Figure 1: Nonexistence of standard of reasonable doubt.

$D_{p}=\{\pi \in \Delta(\Omega): \pi(G) \geq p\}$. Now take the belief $\pi_{1} \in \Delta(\Omega)$, and observe that $\pi_{1}(G)<p_{1}$, while at the same time $\mathbb{E}_{\pi_{1}} V(1)>0$, which is an obvious violation of (3). Intuitively, this is because the juror convicts the defendant under the belief $\pi_{1}$ and acquits him under $\pi_{2}$, even though $\pi_{2}(G)>\pi_{1}(G)$. This is because under $\pi_{1}$ she deems much more likely than under $\pi_{2}$ that the defendant committed the crime intentionally, conditional on him being guilty.

Remark 1. Observe that whenever the juror reasons about events outside $\mathcal{G}$, the set of utility functions such that $V(x)$ is $\mathcal{G}$-measurable is Lebesgue-null. Therefore, generically, a standard of reasonable doubt exists if and only if the juror reasons only about events in $\mathcal{G}$.

$\triangleleft$

\section{Towards resolving the impossibility result}

The previous unexpected result suggests that perhaps the reasonable doubt criterion is too strong to always provide a well-defined decision rule for the juror. This may explain why it has received extensive criticism in the literature, based both on legal arguments but also on empirical evidence. Thus, in this section we attempt to minimally relax it.

Definition 2. Fix an arbitrary $\mathcal{R}$-measurable $U: \Omega \rightarrow \mathbb{R}^{X}$ and an arbitrary $x \in X_{+}$. Then, we say that the pair $\left(p_{x}^{\ell}, p_{x}^{u}\right) \in \mathbb{R}_{+}^{2}$ satisfies the weak reasonable doubt criterion for the sentence $x \in X_{+}$if

(a) $p_{x}^{\ell}$ is the maximum $p \in \mathbb{R}_{+}$such that $C_{x} \subseteq D_{p}$,

(b) $p_{x}^{u}$ is the minimum $p \in \mathbb{R}_{+}$such that $C_{x} \supseteq D_{p}$.

If $p_{x}^{\ell}$ (resp., $p_{x}^{u}$ ) is unique, we refer to it as the lower standard of reasonable doubt (resp., the upper standard of reasonable doubt). 
That is, $p_{x}^{\ell}$ is the largest threshold such that

$$
\pi(G) \leq p_{x}^{\ell} \Rightarrow \mathbb{E}_{\pi} U(x) \leq \mathbb{E}_{\pi} U(0)
$$

i.e., the juror acquits the defendant when the probability that she attaches to him being guilty is below $p_{x}^{\ell}$, but does not necessarily convict him if the probability is larger than $p_{x}^{\ell}$. In this sense, (a) postulates a sufficient condition for acquittance. On the other hand, $p_{x}^{u}$ is the lowest threshold such that

$$
\pi(G) \geq p_{x}^{u} \Rightarrow \mathbb{E}_{\pi} U(x) \geq \mathbb{E}_{\pi} U(0)
$$

i.e., the juror convicts the defendant when the probability that she attaches to him being guilty is above $p_{x}^{u}$, but does not necessarily acquit him if the probability is smaller than $p_{x}^{u}$. In this sense, (b) postulates a sufficient condition for conviction.

Proposition 2. Fix a nontrivial $x \in X_{+}$.

(i) There exist a lower standard of reasonable doubt $p_{x}^{\ell} \in(0,1)$.

(ii) There exist an upper standard of reasonable doubt $p_{x}^{u} \in(0,1]$ if and only if $V_{\omega}(x) \geq 0$ for every $\omega \in G$.

The previous result implies that a sufficient condition for acquittal always exists. On the other hand, a sufficient condition for conviction exists if and only if the juror prefers to always convict a guilty defendant, irrespective of the circumstances under which the crime could have been committed. The latter could also provide a possible justification of why the death penalty has been abolished by most countries, e.g., if it is preferred to acquit a guilty defendant who committed the crime unintentionally over punishing him to death, there is no upper standard of reasonable doubt. Let us provide a graphical illustration of the two weak standards of reasonable doubt.

Example 2 (cont). Recall the example from the previous section (Figure 2.a), observing that $V_{\omega}(1) \geq 0$ for every $\omega \in G$. Consider $p_{1}^{\ell}=1 / 4$ and $p_{1}^{u}=2 / 3$. The area above the lower dashed linear segment contains the beliefs in $D_{1 / 4}=\{\pi \in \Delta(\Omega): \pi(G) \geq 1 / 4\}$, while the area above the upper dashed linear segment contains the beliefs in $D_{1 / 4}=\{\pi \in \Delta(\Omega): \pi(G) \geq 2 / 3\}$. First observe that $D_{2 / 3} \subseteq C_{1} \subseteq D_{1 / 4}$, thus implying that whenever $\pi(G) \leq 1 / 4$ the juror will (weakly) prefer to acquit the defendant, whereas whenever $\pi(G) \geq 2 / 3$ the juror will (weakly) prefer to convict the defendant. Still if $1 / 4<p<2 / 3$, the juror could go either way. Indeed, for every $p>1 / 4$, there will exist some belief $\pi \in \Delta(\Omega)$ belonging to $C_{1}$ but not to $D_{p}$, i.e., the juror would convict the defendant even though $\pi(G)$ would be below $p$. Likewise, for every $p<2 / 3$, there will exist some belief $\pi \in \Delta(\Omega)$ 


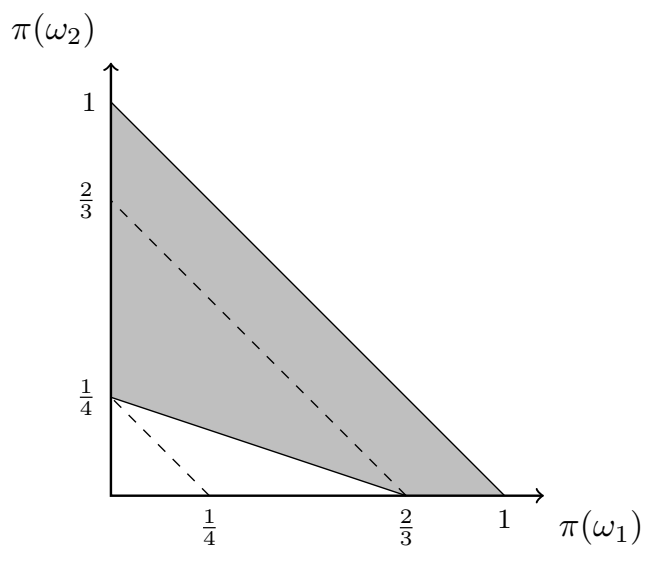

(a) $V_{\omega}(x) \geq 0$ for all $\omega \in G$.

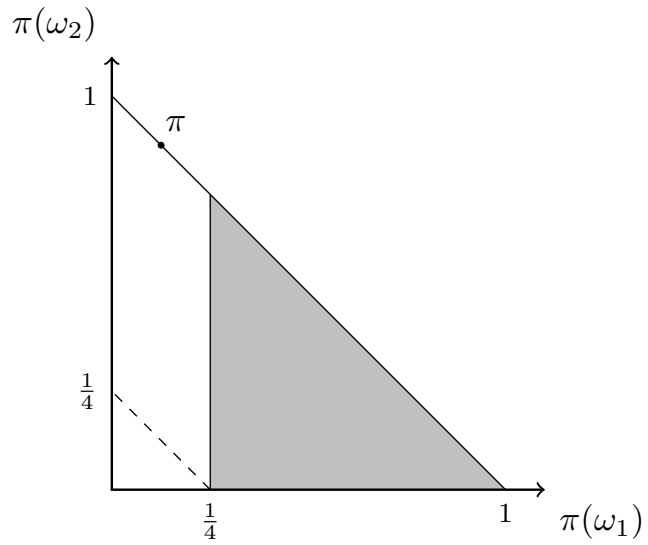

(b) $V_{\omega}(x)<0$ for some $\omega \in G$.

Figure 2: Existence of weak standards of reasonable doubt.

belonging to $D_{p}$ but not to $C_{1}$, i.e., the juror would acquit the defendant even though $\pi(G)$ would be larger than $p$. Hence, the pair $\left(p_{1}^{\ell}, p_{1}^{u}\right)=(1 / 4,2 / 3)$ satisfies the weak reasonable doubt criterion.

Now, let us switch our attention to Figure 2.b, where the state-dependent utility function is such that $U_{\omega_{1}}(x)=-x^{2}-4 x, U_{\omega_{2}}(x)=-x$ and $U_{\omega_{3}}(x)=-x-1$. Notice that there exists some $\omega \in G$ such that $V_{\omega}(1)<0$, viz., $V_{\omega_{2}}(1)=-1$. Similarly to our analysis of Figure 2.a, $p_{1}^{\ell}=1 / 4$ is the lower standard of reasonable doubt. However, notice that there is no upper standard of reasonable doubt, i.e., there is no $p \in(0,1]$ such that $C_{1} \supseteq D_{p}$. Indeed, even if we set $p=1$, the juror may still acquit the defendant, viz., $\pi$ attaches probability 1 to $G$, and still $\mathbb{E}_{\pi} V(1)<0$.

Proposition 3. Fix some nontrivial $x \in X_{+}$such that $V_{\omega}(x) \geq 0$ for all $\omega \in G$. Then, $p_{x}^{\ell} \leq p_{x}^{u}$. Moreover, there exists a standard of reasonable doubt $p_{x} \in(0,1)$ if and only if $p_{x}^{\ell}=p_{x}^{u}$.

The previous result directly suggests that, generically, the lower standard of reasonable doubt will differ from the upper one.

\section{Discussion}

\subsection{Empirical implications}

There is extensive applied research on reasonable doubt. Large part of this literature aims at measuring the standard of reasonable doubt (e.g., Nagel, 1979; Dane, 1985; DeKay, 2006; Wright and Hall, 2007; Dhami, 2008). In particular, different ways to elicit a juror's standard of reasonable doubt have been proposed, such as for instance direct questioning or the utility-based approach, just to mention a few. Not surprisingly, when comparing the estimates induced by the different approaches, 
we find them to differ significantly from each other, e.g., direct questioning typically yields a standard of approximately 0.90 , whereas the utility-based approach induces a standard of approximately $0.50-0.60$ (Connolly, 1987, p.110). There are competing explanations for this discrepancy, focusing either on the juror's inability to understand the concept (Tribe, 1971), or on the existence of framing effects in general (Connolly, 1987).

In the light of our Main Impossibility Theorem, we propose a third explanation, namely that the concept of reasonable doubt is not well-defined. To make our point clearer, first observe that the entire literature on reasonable doubt - explicitly or implicitly - assumes that the juror reasons only about the events in $\mathcal{G}$. In this respect, the discrepancy in the empirical observations may be attributed to the - quite likely - scenario the juror reasons about events outside $\mathcal{G}$, in which case the standard of reasonable doubt does not even exist. Of course, in such a case, it would be unclear what the experimental subject reports when asked to specify her "standard of reasonable doubt". One possibility, is that the subject reports a probability in the interval $\left[p_{x}^{\ell}, p_{x}^{u}\right]$. This would actually justify differences between elicitation methods, as well as differences between estimates that use the same elicitation method, even if the utility functions are assumed to be constant. Overall, we strongly believe that this is an important open question for future research.

\subsection{Objectively null events}

It is quite often the case that during the trial some event is proven to be wrong. Formally, this means that for some $E \in \mathcal{R}$ it becomes transparent that $\pi(E)=0$. Let us refer to this family of events as objectively null. Obviously, when $E$ is objectively null, it does not make sense to even consider beliefs that attach positive probability to $E$ when testing for the reasonable doubt criterion (resp., for the weak weak reasonable doubt criterion). The question is whether in such a case our analysis would still remain valid. It is quite straightforward that our model can be easily modified by replacing the set of $\mathcal{R}$-measurable probability measures with the set of $\mathcal{R}$-measurable conditional probability measures, given $E$. In practice, we would replace the events $C_{x}$ and $D_{p}$ with

$$
\begin{aligned}
& C_{x}(E):=\left\{\pi \in \Delta(\Omega, \mathcal{R}): \mathbb{E}_{\pi} V(x \mid E) \geq 0\right\} \\
& D_{p}(E):=\{\pi \in \Delta(\Omega, \mathcal{R}): \pi(G \mid E) \geq p\}
\end{aligned}
$$

respectively, where $\mathbb{E}_{\pi} V(x \mid E)=\sum_{P \in \mathcal{P}} \pi(P \mid E) V_{P}(x)$, and the reasonable doubt criterion would be satisfied whenever $C_{x}(E)=D_{p}(E)$, and likewise for its weak version. To see that this is the case, consider the measurable subspace $\left(E, \mathcal{G}_{E}\right)$ where $\mathcal{G}_{E}:=\{E, G \cap E, I \cap E, \emptyset\}$, together with the function $V_{\cdot \mid E}(x): E \rightarrow \mathbb{R}$, defined by $V_{\omega \mid E}(x)=V_{\omega}(x)$ for all $\omega \in E$. Then, our Main Impossibility Theorem states that, there exists some $p_{x}$ satisfying the reasonable doubt criterion if and only if $V_{\cdot \mid E}(x)$ is 
$\mathcal{G}_{E}$-measurable. Finally, it is quite straightforward to see that if the reasonable doubt criterion is satisfied (by some $p_{x}$ ) without $E$ being objectively null, it will also be satisfied (perhaps for some other $p_{x}^{\prime}$ ) when $E$ is objectively null. The converse is not necessarily true, i.e., it could be the case that there exists some $p_{x}^{\prime}$ satisfying the reasonable doubt criterion when $E$ is null and at the same time no $p_{x}$ satisfies the reasonable doubt criterion when $E$ is not null. This is for instance the case in Example 1 if we set $E=\left\{\omega_{1}\right\}$.

\subsection{Choosing the sentence}

So far throughout the paper, we have considered cases where the juror makes a binary choice, viz., to convict or to acquit the defendant, with the sentence that follows the conviction verdict being some exogenously given $x \in X_{+}$. However, it is often the case that the juror can choose from a larger set of alternatives $Y \subseteq X$, which still contains 0 , e.g., when there are extenuating circumstances, the juror may decide to choose a reduced sentence. For simplicity, let $Y$ be compact and the utility function $U$ be continuous. Then, for a given frame $\mathcal{R}$, the juror chooses the mostpreferred sentence $x^{\pi}:=\max _{x \in Y} \mathbb{E}_{\pi} U(x)$ for every $\pi \in \Delta(\Omega, \mathcal{R})$. The fact that $Y$ is compact and $U$ continuous guarantees that there exists an optimal sentence for every $\pi \in \Delta(\Omega, \mathcal{R})$. Obviously, $x^{\pi}$ may differ across different beliefs. Now, the question is whether such flexibility in choosing the sentence could guarantee the existence of a standard of reasonable doubt. In particular, is there some $p \in \mathbb{R}_{+}$such that $\left\{\pi \in \Delta(\Omega, \mathcal{R}): \max _{x \in Y} \mathbb{E}_{\pi} V(x) \geq 0\right\}=: C_{Y}=D_{p}$ ? The answer turns out to be negative, even in cases where $V(x)$ is $\mathcal{G}$-measurable for every $x \in Y$. Intuitively, the reason is that $C_{Y}$ is convex, but does not necessarily coincide with the intersection of a half-space with $\Delta(\Omega, \mathcal{R})$, i.e., $\pi \mapsto \max _{x \in Y} \mathbb{E}_{\pi} V(x)$ is not necessarily affine, whereas $\pi \mapsto \pi(G)$ is. Hence, our Impossibility Theorem does not rely on $x$ being exogenously specified.

\section{A. Proofs}

Proof of Proposition 1. (i) Sufficiency. Let $p_{x} \in(0,1)$ satisfy the reasonable doubt criterion for $x \in X_{+}$, and suppose - contrary to what we want to show - that $x$ is trivial. Then, there are two possible (not mutually exclusive) cases that we consider:

(a) Let $V(x) \geq 0$. In this case, $\mathbb{E}_{\pi} V(x) \geq 0$ for every $\pi \in \Delta(\Omega, \mathcal{R})$. Moreover, by $p_{x}>0$, it follows that there exists some $\rho \in \Delta(\Omega, \mathcal{R})$ such that $\rho(G)<p_{x}$. Hence, $\Delta(\Omega, \mathcal{R})=C_{x}=D_{p_{x}} \subsetneq \Delta(\Omega, \mathcal{R})$, which is an obvious contradiction.

(b) Let $V(x) \leq 0$. In this case, $\mathbb{E}_{\pi} V(x) \leq 0$ for every $\pi \in \Delta(\Omega, \mathcal{R})$, with equality holding if and only if $V_{P}(x)=0$ for all $P \in \mathcal{P}$ with $\pi(P)>0$. Hence, $D_{p_{x}}=C_{x}=\left\{\pi \in \Delta(\Omega, \mathcal{R}): \mathbb{E}_{\pi} V(x)=0\right\}$. Now, 
consider three subcases:

(b.1) Let $P \subseteq G \Leftrightarrow V_{P}(x)=0$. Then, $\{\pi \in \Delta(\Omega, \mathcal{R}): \pi(G)=1\}=\left\{\pi \in \Delta(\Omega, \mathcal{R}): \mathbb{E}_{\pi} V(x)=0\right\}$, implying that $p_{x}=1$ which is a contradiction.

(b.2) Let $V_{P}(x)<0$ for some $P \subseteq G$. Consider $\rho \in \Delta(\Omega, \mathcal{R})$ such that $\rho(P)=1$. Then, $\rho \in\{\pi \in$ $\left.\Delta(\Omega, \mathcal{R}): \pi(G) \geq p_{x}\right\}$ and yet $\rho \notin\left\{\pi \in \Delta(\Omega, \mathcal{R}): \mathbb{E}_{\pi} V(x)=0\right\}$, which is again a contradiction.

(b.3) Let $V_{P}(x)=0$ for some $P \in \mathcal{P}$ with $P \cap G=\emptyset$. Consider $\rho \in \Delta(\Omega, \mathcal{R})$ such that $\rho(P)=1$. Then, $\rho \notin\left\{\pi \in \Delta(\Omega, \mathcal{R}): \pi(G) \geq p_{x}\right\}$ and yet $\rho \in\left\{\pi \in \Delta(\Omega, \mathcal{R}): \mathbb{E}_{\pi} V(x)=0\right\}$, which is again a contradiction.

Thus, we conclude that $x$ is nontrivial.

Necessity. Let $x \in X_{+}$be nontrivial, thus implying that the $\mathcal{R}$-measurable events $V^{+}(x):=\{\omega \in \Omega$ : $\left.V_{\omega}(x)>0\right\}$ and $V^{-}(x):=\left\{\omega \in \Omega: V_{\omega}(x)<0\right\}$ are both nonempty. Now, suppose - contrary to what we want to prove - that $p_{x} \notin(0,1)$. Then, there are two possible cases that we consider:

(a) Let $p_{x} \leq 0$. Then, we obtain $\Delta(\Omega, \mathcal{R})=D_{p_{x}}=C_{x}$, which is a contradiction, as the probability measure $\rho \in \Delta(\Omega, \mathcal{R})$ with $\rho\left(V^{-}(x)\right)=1$ does not belong to $\left\{\pi \in \Delta(\Omega, \mathcal{R}): \mathbb{E}_{\pi} V(x) \geq 0\right\}$.

(b) Let $p_{x} \geq 1$, thus implying that there are two subcases:

(b.1) Let $p_{x}>1$. In this case we obtain $\emptyset=D_{p_{x}}=C_{x}$, thus implying that $\mathbb{E}_{\pi} V(x)<0$ for all $\pi \in$ $\Delta(\Omega, \mathcal{R})$. But then since $V^{+}(x) \neq \emptyset$, the probability measure $\rho \in \Delta(\Omega, \mathcal{R})$ with $\rho\left(V^{+}(x)\right)=1$ is such that $E V_{\rho}(x)>0$ which is a contradiction.

(b.2) Let $p_{x}=1$ in which case $C_{X}=D_{p_{x}}=\{\pi \in \Delta(\Omega, \mathcal{R}): \pi(G)=1\}$. Now suppose that $G \cap V^{-}(x) \neq \emptyset$ and take $\rho \in \Delta(\Omega, \mathcal{R})$ such that $\rho\left(G \cap V^{-}(x)\right)=1$, implying that $\rho(G)=1$. But then observe that $E V_{\rho}(x)<0$, which is a contradiction. Therefore, it must necessarily be the case that $G \cap V^{-}(x)=\emptyset$. Now take arbitrary $P \in V^{+}(x)$ and $Q \in V^{-}(x)$, and for every $\lambda \in[0,1]$ consider the probability measure $\mu_{\lambda} \in \Delta(\Omega, \mathcal{R})$ such that $\mu_{\lambda}(P)=\lambda$ and $\mu_{\lambda}(Q)=1-\lambda$. By continuity there exists some $\tilde{\lambda} \in(0,1)$ such that $E V_{\mu_{\tilde{\lambda}}}(x)=\tilde{\lambda} V_{P}(x)+(1-\tilde{\lambda}) V_{Q}(x) \geq 0$. But then $\mu_{\tilde{\lambda}} \in\left\{\pi \in \Delta(\Omega, \mathcal{R}): \mathbb{E}_{\pi} V(x) \geq 0\right\}$ and at the same time $\mu_{\tilde{\lambda}}\left(V^{-}(x)\right)>0$, which by the fact that $G \cap V^{-}(x)=\emptyset$ implies that $\mu_{\tilde{\lambda}} \notin\{\pi \in \Delta(\Omega, \mathcal{R}): \pi(G)=1\}$. The latter is a contradiction.

(ii) Contrary to what we want to show, suppose that there exists some $\tilde{p}_{x} \neq p_{x}$ satisfying the reasonable doubt criterion. By part (i) both of them belong to $(0,1)$, and without loss of generality let $0<\tilde{p}_{x}<p_{x}<1$. Then, $C_{x}=D_{p_{x}} \subsetneq D_{\tilde{p}_{x}}=C_{x}$ which is an obvious contradiction.

Proof of Main Theorem. Necessity. Fix some $\mathcal{R}$-measurable $U: \Omega \rightarrow \mathbb{R}^{X}$ and take some $p_{x} \in(0,1)$ satisfying the reasonable doubt criterion. Begin by identifying $\Delta(\Omega, \mathcal{R})$ with the unit simplex over the finite set $\mathcal{P}$, which spans the hyperplane

$$
H=\left\{q \in \mathbb{R}^{\mathcal{P}}: \sum_{P \in \mathcal{P}} q(P)=1\right\}
$$


of the $|\mathcal{P}|$-dimensional euclidean space. Now take the hyperplanes of $H$,

$$
\begin{aligned}
H_{c} & :=\left\{q \in H: \sum_{P \in \mathcal{P}} q(P) V_{P}(x)=0\right\} \\
H_{d} & :=\left\{q \in H: \sum_{P \in \mathcal{P}} q(P) W_{P}(x)=p_{x}\right\} \\
& =\left\{q \in H: \sum_{P \in \mathcal{P}} q(P) W_{P}(x)=\sum_{P \in \mathcal{P}} q(P) p_{x}\right\} \\
& =\left\{q \in H: \sum_{P \in \mathcal{P}} q(P)\left(W_{P}(x)-p_{x}\right)=0\right\} .
\end{aligned}
$$

where $W_{P}(x):=1$ if $P \subseteq G$ and $W_{P}(x):=0$ otherwise. Notice that both $H_{c}$ and $H_{d}$ are the hyperplanes associated with the half-spaces $C_{x}$ and $D_{p_{x}}$ respectively. Hence, by the fact that $C_{x}=D_{p_{x}}$ it follows that $H_{c}=H_{d}$. Thus, there exists some $\lambda \in \mathbb{R}$ such that $V_{P}(x)=\lambda\left(W_{P}(x)-p_{x}\right)$ for all $P \in \mathcal{P}$. That is, $V_{P}(x)=\lambda\left(1-p_{x}\right)$ for each $P \subseteq G$ and $V_{P}(x)=-\lambda p_{x}$ for each $P \subseteq I$. Therefore $V(x)$ is $\mathcal{G}$-measurable.

Sufficiency. Fix some $\mathcal{R}$-measurable $U: \Omega \rightarrow \mathbb{R}^{X}$ such that $V(x)$ is $\mathcal{G}$-measurable. Define $V_{G}(x):=$ $V_{P}(x)$ for $P \subseteq G$ and $V_{I}(x):=V_{P}(x)$ for $P \subseteq I$. Notice that by Assumption $1, V_{I}(x) \leq 0$. In fact, since $x$ is nontrivial, we obtain $V_{I}(x)<0$ and $V_{G}(x)>0$. Then,

$$
\begin{aligned}
C_{x} & =\left\{\pi \in \Delta(\Omega, \mathcal{R}): \sum_{P \in \mathcal{P}} \pi(P) V_{P}(x) \geq 0\right\} \\
& =\left\{\pi \in \Delta(\Omega, \mathcal{R}): \pi(G) V_{G}(x)+(1-\pi(G)) V_{I}(x) \geq 0\right\} \\
& =\left\{\pi \in \Delta(\Omega, \mathcal{R}): \pi(G)\left(V_{G}(x)-V_{I}(x)\right) \geq-V_{I}(x)\right\} \\
& =\left\{\pi \in \Delta(\Omega, \mathcal{R}): \pi(G) \geq V_{I}(x) /\left(V_{I}(x)-V_{G}(x)\right)\right\} .
\end{aligned}
$$

Thus, $p_{x}:=V_{I}(x) /\left(V_{I}(x)-V_{G}(x)\right)$ satisfies the reasonable doubt criterion, and obviously $p_{x} \in(0,1)$ since $V_{I}(x)<0$ and $V_{G}(x)>0$, thus completing the proof.

Proof of Proposition 2. We identify $\Delta(\Omega, \mathcal{R})$ with the unit simplex over the finite set $\mathcal{P}$ which spans the hyperplane $H$ of the $|\mathcal{P}|$-dimensional euclidean space $\mathbb{R}^{\mathcal{P}}$, as in the proof of the Main Theorem. For any convex set $M \subseteq H$, let $\delta^{*}(\cdot \mid M): H \rightarrow \mathbb{R}$ be the support function of $M$, defined by

$$
\delta^{*}(q \mid M):=\sup \left\{\sum_{P \in \mathcal{P}} \pi(P) q(P) \mid \pi \in M\right\}
$$

for each $q \in H \subseteq \mathbb{R}^{\mathcal{P}}$. Then, it follows from Rockafellar (1970, p.112) that, for every $q \in H$,

$$
M \subseteq\left\{\pi \in H: \sum_{P \in \mathcal{P}} \pi(P) q(P) \leq p\right\} \Leftrightarrow p \geq \delta^{*}(q \mid M)
$$

(i) Set $M:=C_{x}$ and $q=-W(x)$, where recall that $W_{P}(x)=1$ if $P \subseteq G$ and $W_{P}(x)=0$ if $P \subseteq I$, and define $p_{x}^{\ell}:=-\delta^{*}\left(-W(x) \mid C_{x}\right)$. Hence, by (A.1) it follows that

$$
\begin{aligned}
C_{x} \subseteq\{\pi \in \Delta(\Omega, \mathcal{R}): \pi(G) \geq p\} & \Leftrightarrow C_{x} \subseteq\{\pi \in \Delta(\Omega, \mathcal{R}):-\pi(G) \leq-p\} \\
& \Leftrightarrow-p \geq-p_{x}^{\ell} \\
& \Leftrightarrow p \leq p_{x}^{\ell} .
\end{aligned}
$$


Now, contrary to what we want to prove, assume $p_{x}^{\ell} \geq 0$. Then, there exists some $\rho \in\left\{\pi \in C_{x}: \pi(G)<p\right\}$ for every $p>0$. However, by Assumption 1 , if $\pi(G)=0$ then $\mathbb{E}_{\pi} V(x)<0$, and by continuity of $U$, there exists some $\varepsilon>0$ such that $\mathbb{E}_{\pi} V(x)<0$ for all $\pi \in \Delta(\Omega, \mathcal{R})$ with $\pi(G)<\varepsilon$. Hence, by setting $p:=\varepsilon$ we reach is a contradiction. Therefore, $p_{x}^{\ell}>0$. Finally, contrary to what we want to show, assume that $p_{x}^{\ell} \geq 1$. Then, $C_{x} \subseteq\{\pi \in \Delta(\Omega, \mathcal{R}): \pi(G) \geq p\}$ for all $p \leq 1$, implying that $C_{x} \subseteq\{\pi \in \Delta(\Omega, \mathcal{R}): \pi(G)=1\}$. However, by continuity, this can only be the case if $V_{P}(x)=0$ for all $P \subseteq G$. But then, it is the case that $V(x) \leq 0$, i.e., $x$ is trivial, which is a contradiction. Hence, $p_{x}^{\ell}$ is the lower standard of reasonable doubt.

(ii) Define the convex set $A_{x}:=\left\{\pi \in \Delta(\Omega, \mathcal{R}): \mathbb{E}_{\pi} V(x)<0\right\}=\Delta(\Omega, \mathcal{R}) \backslash C_{x}$. Set $q=W(x)$, and let $p_{x}^{u}:=\delta^{*}\left(W(x) \mid A_{x}\right)$. Hence,

$$
\begin{aligned}
C_{x} \supseteq\{\pi \in \Delta(\Omega, \mathcal{R}): \pi(G) \geq p\} & \Leftrightarrow C_{x} \supseteq\{\pi \in \Delta(\Omega, \mathcal{R}): \pi(G)>p\} \\
& \Leftrightarrow A_{x} \subseteq\{\pi \in \Delta(\Omega, \mathcal{R}): \pi(G) \leq p\} \\
& \Leftrightarrow p \geq p_{x}^{u},
\end{aligned}
$$

with the first equivalence following from the fact that $C_{x}$ is closed and $\{\pi \in \Delta(\Omega, \mathcal{R}): \pi(G) \geq p\}=\operatorname{clos}(\{\pi \in$ $\Delta(\Omega, \mathcal{R}): \pi(G)>p\})$. Now, contrary to what we want to prove, let $p_{x}^{u} \leq 0$. Then, $C_{x} \supseteq\{\pi \in \Delta(\Omega, \mathcal{R}):$ $\pi(G) \geq p\}$ for all $p \geq 0$, implying $C_{x} \supseteq\{\pi \in \Delta(\Omega, \mathcal{R}): \pi(G) \geq 0\}=\Delta(\Omega, \mathcal{R})$, which contradicts Assumption 1. Finally, let us prove that $p_{x}^{u} \leq 1$ if and only if $V_{P}(x) \geq 0$ for all $P \subseteq G$. We begin with sufficiency. Contrary to what we want to show, let $p_{x}^{u}>1$, thus implying that $C_{x} \nsupseteq\{\pi \in \Delta(\Omega, \mathcal{R}): \pi(G) \geq 1\}$, i.e., there exists some $\pi \in \Delta(\Omega, \mathcal{R})$ with $\pi(G)=1$ such that $\mathbb{E}_{\pi} V(x)<0$. But this contradicts our hypothesis that $V_{P}(x) \geq 0$ for all $P \subseteq G$. Finally we prove necessity. Contrary to what we want to show let $V_{P}(x)<0$ for some $P \subseteq G$. But then, observe that $1 \geq p_{x}^{u}$ and at the same time $C_{x} \nsupseteq\{\pi \in \Delta(\Omega, \mathcal{R}): \pi(G) \geq 1\}$, which is a contradiction. To see this, take some $\pi \in \Delta(\Omega, \mathcal{R})$ such that $\pi(P)=1$, which yields $\mathbb{E}_{\pi} V(x)<0$.

Proof of Proposition 3. It follows from Proposition 2 that $D_{p_{x}^{u}} \subseteq C_{x} \subseteq D_{p_{x}^{\ell}}$. Hence, $p_{x}^{u} \geq p_{x}^{\ell}$. Now, let us prove sufficiency for our second claim. If $p_{x}^{\ell}=p_{x}^{u}=: p_{x}$, then $D_{p_{x}^{u}}=D_{p_{x}^{\ell}}=D_{p_{x}}$. But, then since $D_{p_{x}} \subseteq C_{x} \subseteq D_{p_{x}}$, it must be the case that $C_{x}=D_{p_{x}}$, implying that $p_{x}$ is the standard of reasonable doubt. Finally, let us prove necessity for our second claim. Suppose contrary to what we want to prove that $p_{x}^{\ell}<p_{x}^{u}$. This implies that $D_{p_{x}^{u}} \subsetneq D_{p_{x}^{\ell}}$, and therefore either $D_{p_{x}^{u}} \subsetneq C_{x}$ or $C_{x} \subsetneq D_{p_{x}^{\ell}}$. This implies that there is no $p$ such that $C_{x}=D_{p}$, which is a contradiction.

\section{References}

Ahn, D. \& Ergin, H. (2010). Framing contingencies. Econometrica 78, 655-695.

Andreoni, J. (1991). Reasonable doubt and the optimal magnitude of fines: should the penalty fit the crime? RAND Journal of Economics 22, 385-395. 
Becker, G.S. (1968). Crime and punishment: an economic approach. Journal of Political Economy 76, $169-217$.

Connolly, T. (1987). Decision theory, reasonable doubt, and the utility of erroneous acquittals. Law and Human Behavior 11, 101-112.

DANE, F.C. (1985). In search of reasonable doubt: a systematic examination of selected quantification approaches. Law and Human Behavior 9, 141-158.

De Oliveira, H., Denti, T., Minm, M. \& Ozbek, K. (2016). Rationally inattentive preferences and hidden information costs. Theoretical Economics, forthcoming.

DeKay, M.L. (2006). The difference between the Blackstone-like error ratios and probabilistic standards of proof. Law and Social Inquiry 21, 95-132.

Dhami, M.K. (2008). On measuring quantitative interpretations of reasonable doubt. Journal of Experimental Psychology: Applied 14, 353-363.

Diamond, H.A. (1990). Reasonable doubt: to define or not to define. Columbia Law Review 90, 1716-1736.

Ergin, H. \& SARver, T. (2010). A unique costly contemplation representation. Econometrica 78, 12851339.

Gerardi, D. (2000). Jury verdicts and preference diversity. American Political Science Review 94, 395-406.

Feddersen, T. \& Pesendorfer, W. (1998). Convicting the innocent: the inferiority of unanimous jury verdicts under strategic voting. American Political Science Review 92, 23-35.

Fishburn, P.C. (1973). A mixture-set axiomatization of conditional subjective expected utility Econometrica $41,1-25$.

Friedman, E. \& Wickelgren, A.L. (2006). Bayesian juries and the limits to deterrence. Journal of Law, Economics and Organization 22, 70-86.

Jonakait, R.N. (2003). The American jury system. Yale University Press.

Kamenica, E. \& Gentzkow, M. (2011). Bayesian persuasion. American Economic Review 101, 25902615.

-(2014). Costly persuasion. American Economic Review: Papers $\&$ Proceedings 104, 457-462.

KARni, E. (1993a). A definition of subjective probabilities with state-dependent preferences. Econometrica $61,187-198$. 
- (1993b). Subjective expected utility with state-dependent preferences. Journal of Economic Theory 60, 428-438.

Karni, E., Schmeidler, D. \& Vind, K. (1983). On state dependent preferences and subjectives probabilities. Econometrica 51, 1021-1031.

LiLlquist, E. (2002). Recasting reasonable doubt: decision theory and the virtues of variability. U.C. Davis Law Review 86, 85-197.

Mulrine, T.V. (1997). Reasonable doubt: how in the world is it defined? American University International Law Review 12, 195- 225.

NAGEL, S. (1979). Bringing the values of the jurors in line with the law. Judicature 63, 189-195.

Persson, M. \& Siven, C.H. (2007). The Becker paradox and type I versus type II errors in the economics of crime. International Economic Review 48, 211-233.

Rockafellar, R.T. (1970). Convex analysis. Princeton University Press.

Tribe, L.H. (1971). Trial by mathematics: precision and ritual in the legal process. Harvard Law Review 84, 1329-1393.

Wright, D.B. \& Hall, M. (2007). How a "reasonable doubt" instruction affects decisions of guilt. Basic and Applied Social Psychology 29, 91-98. 